\title{
Modal Analysis of Splitter Fixed Structure in Concentrator-Splitter Optics System
}

\author{
Bo $\mathrm{Li}^{1, \mathrm{a}^{*}}$,Suping Bai ${ }^{1, \mathrm{~b}}$, Yan $\mathrm{An}^{2, \mathrm{c}}$ \\ ${ }^{1}$ Changchun University of Science and Technology, Changchun130022, China \\ ${ }^{2}$ Institute of Space Photoelectric Technology,Changchun University of Science and \\ Technology, Changchun 130022, China \\ a18843166649@163.com, bbaisp@126.com, anyan_7@126.com
}

Keyword: Concentrator-Splitter Optics System; splitter; modal analysis; dynamic characteristics Abstract:As the core component of Concentrator-Splitter Optics System, the beam splitter has an important influence on solar energy utility efficiency improvement. The vibration frequency of beam splitter fixed structure caused by external stimulation has great influence on system accuracy. So, it is necessary to analyze the natural frequency and its model shapes. This paper describes the finite element modal analysis theory briefly, establishes analysis model structure of the fixed structure by ABAQUS, obtains each vibration mode shape and its corresponding natural frequency. Finally, we optimize the mechanical structure according to the numerical analysis results and propose a method that install rubber shock pad between the system and protective box to decrease the external stimulation influence on system accuracy. This work has a reference value to ensure the solar energy utility efficiency of the system.

\section{Introduction}

Concentrating solar power can improve the photon-to-electron conversion efficiency of solar cell. Traditional photovoltaic power generations mainly convert the energy near visible spectrum into electricity. But ,the visible spectrum(380nm $760 \mathrm{~nm})$ energy only percents $61.4 \%$ of the whole energy, meanwhile the ultraviolet spectrum energy percents $9.4 \%$, the infrared spectrum energy percents $29.1 \%$. Obviously the traditional photovoltaic power generation device can not make full use of solar energy, especially the infrared spectrum can not only converted into electricity, but also decreases the photoelectric conversion efficiency. So developing photovoltaic / thermal integrated utilization technology is an important research direction. In this paper, we design a Concentrator-Splitter Optics System which its solar energy utility efficiency can reach a high value of $63 \%$, compared with the efficiency less than $50 \%$ of the traditional photovoltaic power generation device, it has a great improvement. The system mainly includes three parts: Fresnel lens, beam splitter and GaAs batteries. The operating principle is shown in Fig.1. The sunlight focus on beam splitter through the Fresnel lens, then divided into 400nm-900nm spectrum and $900 \mathrm{~nm}-1800 \mathrm{~nm}$ by the splitter, finally the two spectrums are received by the photovoltaic receiving 
device and the thermoelectric receiving device to achieve the purpose that utilizing the visible and near-infrared spectrum energy of the sunlight. As the key and high-cost part of the system, beam splitter plays an important role on increasing solar energy utility efficiency significantly. The efficiency can be reduced greatly when the surface shape changed obviously because the two beams can not be fully converged to the receiving end. So it is necessary to analyze the splitter fixed structure's natural frequency and model shapes to guarantee the normal working condition of the mirror. Fig.2. is the beam path diagram.

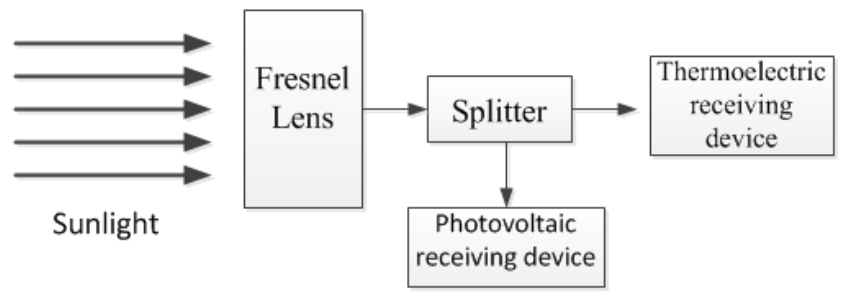

Fig.1. Operating principle of the system

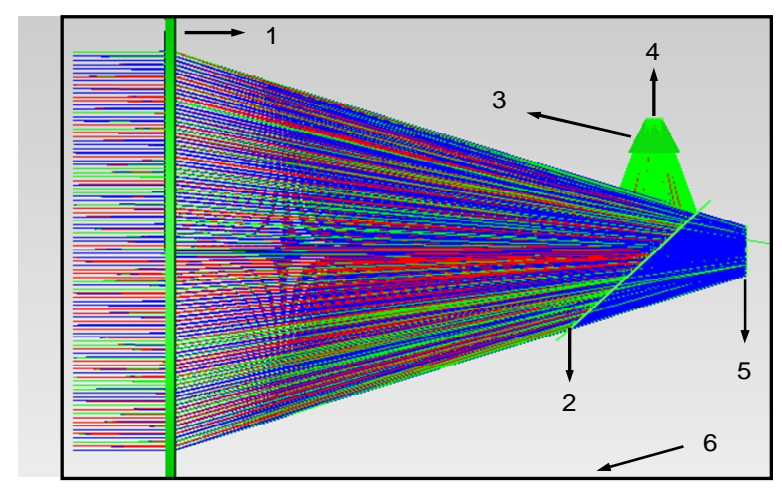

Fig.2 .Beam path diagram

1-Fresnel lens;2-Splitter;3-Light funnel;4-Photovoltaic receiving device interface;

5-thermoelectric receiving device interface;6-mechanical structure

\section{Design of beam splitter fixed structure and modal analysis}

The $120 \mathrm{~mm} \times 120 \mathrm{~mm} \times 1.1 \mathrm{~mm}$ splitter is made of high borosilicate glass, cutoff wavelength is 400-900nm. The high costing manufacture of the splitter needs a complex process of engraving, polishing, ultrasonic cleaning and coating. Meanwhile, in assembly process, it requires the splitter fastened stably and angle fine-tuning to guarantee the two spectrum can focus on receivers sufficiently. Then, we design the structure as follows shown in Fig.3. It consists of five components:guide groove that used for adjusting angle between splitter and horizontal direction, splitter bottom frame for fixing mirror, support plate and splitter base which used for connecting and fixing with the other structures of system, beam splitter used for dividing sunlight into visible spectrum and infrared spectrum. The heat sink and photovoltaic receiving device are installed on support plates. 


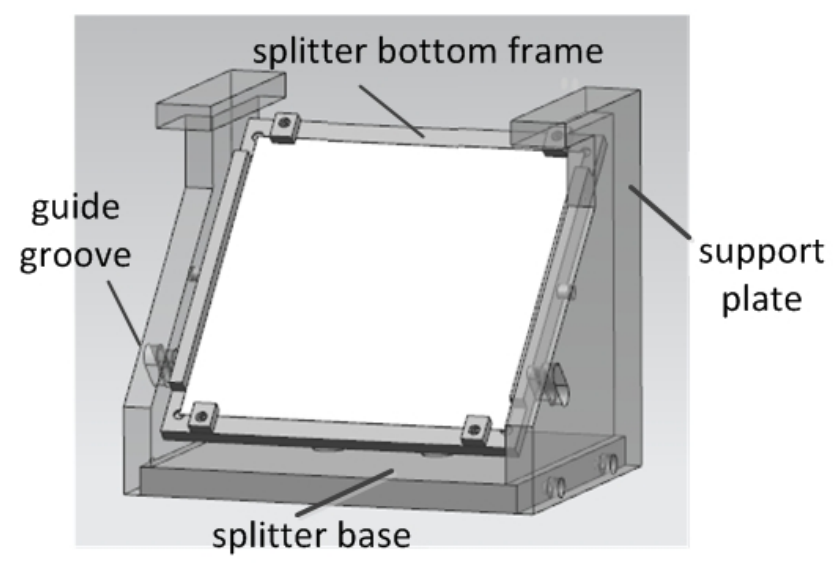

Fig.3. the splitter fixed structure

In practical application, the splitter bottom frame is solidly connected with two support plates.

The splitter is fastened on the bottom frame by pressing blocks and its position will be fixed by the dispensing bolts between the guide groove and bottom frame when debugged correctly. In this condition, we affirm that the bolts will not be loose by any external stimulation. At the same time, we simplify the model to ensure the uniformity of mesh division in modal analysis[2]. We mesh the structure as shown in Fig.4.(a).

The material of the system is $2 \mathrm{~A} 12$ hard aluminum alloys, taking it as linear elastic material in calculation. The elastic modulus of the model E=70GPa, Poisson ratio $\mu=0.3$, density $\rho=2.7 \mathrm{~g} / \mathrm{cm}^{3}$. The simplified model of the fixed structure is imported into ABAQUS software, with material properties, and meshing[3]. In constraint settings, all degrees of freedom of the structure is constraint at its bottom. Eigenvalues and modal reflect the characteristics of structure and frequency. One can any linear structure response from the frequency and vibration mode obtained by modal decomposition method[4]. Typically, considering the first few vibration modes can get considerable accuracy solutions. The magnitude of the vibration modes is only a relative value, which characterizes the relative vibration ratio of a certain first order natural frequency, it reflects the transmission condition of the natural frequency, not the actual vibration values. Through modal analysis, 3 extended natural frequencies and modal shapes of structure are given in Fig.4.(b),(c),(d). The 8 extended natural frequencies is shown in Table 1 . 


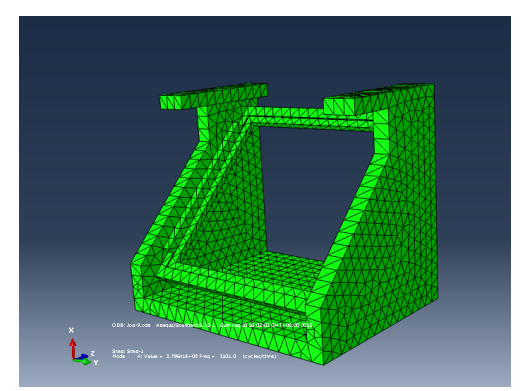

(a)

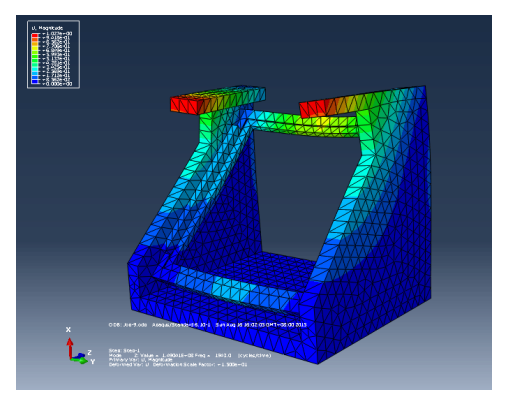

(c)

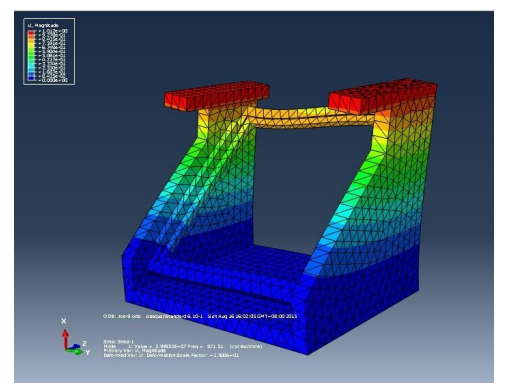

(b)

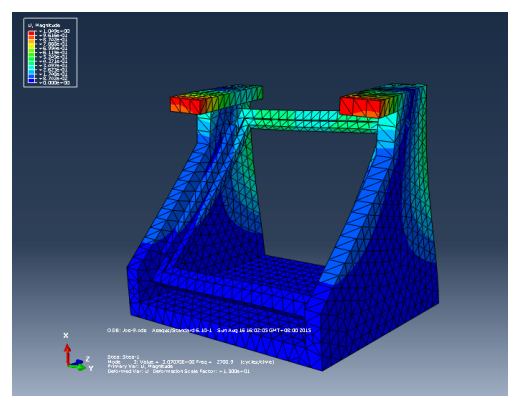

(d)

Fig.4. meshing and modal shapes

(a) meshing model;(b) 1 extended modal shape;

(c) 2 extended shape;(d) 3 extended shape

Table 1 the 8 extended frequencies

\begin{tabular}{|c|c|}
\hline order & Frequency[HZ] \\
\hline 1 & 875.51 \\
\hline 2 & 1943.0 \\
\hline 3 & 2788.9 \\
\hline 4 & 3101.0 \\
\hline 5 & 3271.0 \\
\hline 6 & 3758.5 \\
\hline 7 & 3831.1 \\
\hline 8 & 4004.9 \\
\hline
\end{tabular}

In practical application, the 16 subsystems are assembled together as a whole, and then installed on the large solar tracker which can achieve the accurate tracking after the sun, in this way, to ensure the system efficiency. The solar tracker used in this system is a large-scale instrument which always influenced by severe wind and other heavy weather, its vibration frequency ranges from several ten to $3000 \mathrm{~Hz}$. The analysis results show that the 4 extended frequencies are almost under $3000 \mathrm{~Hz}$, obviously, it's susceptible to arouse resonance and damage the splitter. So it's necessary to optimize the structure to guarantee the splitter working well. 


\section{Optimization design and result analysis}

In the fact of this, we optimize the mechanical structure to limit the DOF of the support plates by installed stiffeners to fix thermoelectric receiver structure and splitter fixed structure together tightly. Then, do the analysis again and obtain the new results shown in Table 2. The new structure 3D model and its modal shape of first order vibration frequency is shown in Fig.5. The 3D model is show in Fig.6. Apparently, the natural frequency has been improved greatly. The frequencies of first two order have already been higher than $3000 \mathrm{~Hz}$. From the third order, the natural frequency is far greater than the working vibration frequency. So the new structure can effectively avoid the external stimulation effect on the accuracy of the system. Finally, we propose a method that install rubber shock pad between the system and protective box to further decrease the natural frequency.

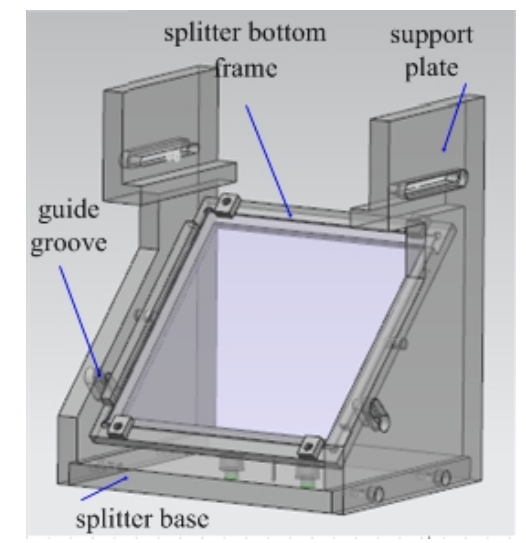

(a)The new structure 3D model

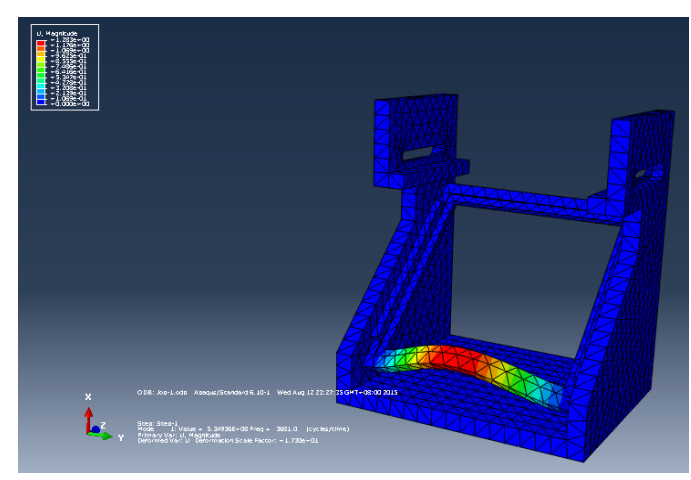

(b) new modal shape of first order

Fig.5.new structure and first order vibration frequency

Table 2.the new 8 extended frequencies

\begin{tabular}{|c|c|}
\hline order & Frequency[HZ] \\
\hline 1 & 3681.0 \\
\hline 2 & 3685.7 \\
\hline 3 & 5246.5 \\
\hline 4 & 5258.8 \\
\hline 5 & 9542.8 \\
\hline 6 & 12935.0 \\
\hline 7 & 12953.3 \\
\hline 8 & 15650.2 \\
\hline
\end{tabular}

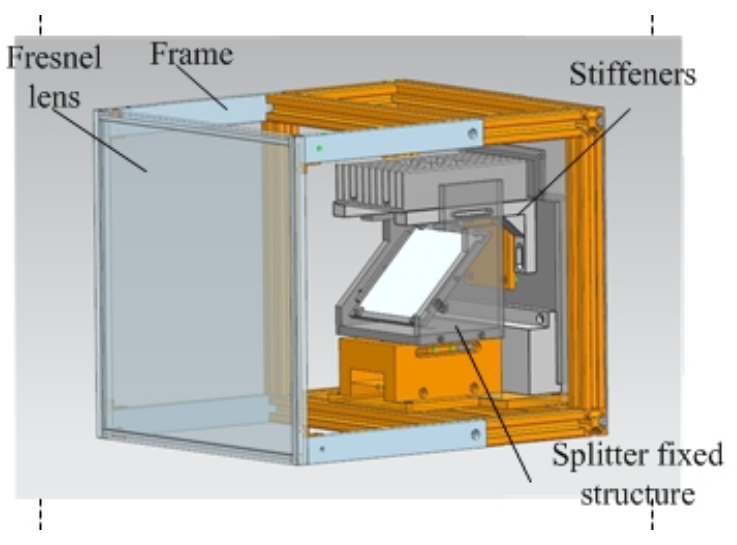

Fig.6. 3D model 


\section{Conclusions}

In this paper, we analyze the natural frequency of the splitter fixed structure and obtain the numerical values, then optimize the existing structure according to requirements of the actual application environment, finally the splitter can be protected efficiently that ensure a preferable system accuracy.

\section{Literature References}

[1] Jufang Sun, Finite Element Method and Its Application, Beijing, 1990.in Chinese

[2] Yanyan Cao, Dengfeng Zhao, Finite Element Modal Analysis Theory and Application,J. Mechanical Engineering and Automation, 140(2007) 73-74.in Chinese

[3] Haiming Gu, Mechanical Vibration Theory and Practical, Beijing, 2007.in Chinese

[4] Xiaoming Ma, Zhenqiang Yao, Finite element analysis of large span portal frame structure,J. Machine Design and Research, 6(2008)10-12.in Chinese 